\title{
A SYMMETRIC DENSITY PROPERTY: MONOTONICITY AND THE APPROXIMATE SYMMETRIC DERIVATIVE
}

\author{
C. FREILING AND D. RINNE
}

(Communicated by R. Daniel Mauldin)

\begin{abstract}
The following is established:
Let $W$ and $B$ be open sets of real numbers whose union has full measure. If for each $x$, the set $\{h>0 \mid x-h \in W, x+h \in B\}$ has density zero at zero, then these sets are all empty.

This is then used to prove the following:

If $f$ is a continuous real valued function with a nonnegative lower approximate symmetric derivative, then $f$ is nondecreasing.
\end{abstract}

Introduction. We will establish the following result.

Let $W$ and $B$ be open subsets of an interval whose union has full measure in this interval. If for each $x$ in the interval, the set $\{h>0 \mid x-h \in W, x+h \in B\}$ has density zero at zero, then these sets are all empty.

We then use this to prove the following.

If $f$ is a continuous real valued function with a nonnegative lower approximate symmetric derivative, then $f$ is nondecreasing.

Early attempts to prove this theorem can be found in [1] and [5]. More recently, some partial results have been established $[3,4,6]$. A survey of this and related topics appears in Larson [2].

DEFINITIONS. We will use the following notation and definitions. All functions are real valued and $\lambda$ is Lebesgue measure on the real line. For a set $A, \chi_{A}(x)$ is the characteristic function of $A$. For $A$ measurable, the upper right density of $A$ at $x$ is $\bar{d}^{+}(A, x)=\lim \sup \lambda(A \cap(x, x+h)) / h$ as $h \rightarrow 0^{+}$. The upper left, lower right, and lower left densities, $\bar{d}^{-}, \underline{d}^{+}$, and $\underline{d}^{-}$are defined in a similar manner. If these four values are the same, their common value is $d(A, x)$, the density of $A$ at $x$. The upper right Dini derivate of $f$ is $D^{+} f(x)=\lim \sup (f(x+h)-f(x)) / h$ as $h \rightarrow 0^{+}$. The upper left, lower right, and lower left derivates, $D^{-} f, D_{+} f$, and $D_{-} f$ are defined similarly. For sets $A$ and $B$, we define $A B(x)=\{h>0 \mid x-h \in A, x+h \in B\}$. The lower approximate symmetric derivative of $f, \underline{f}_{a p}^{(1)}(x)$, is the least upper bound of the set of $\alpha$ such that $\{h \mid(f(x+h)-f(x-h)) / 2 h<\alpha\}$ has density zero at 0 . The upper approximate symmetric derivative, $\bar{f}_{a p}^{(1)}(x)$, is defined similarly. If $\underline{f}_{a p}^{(1)}(x)=$ $\bar{f}_{a p}^{(1)}(x)$, their common value is the approximate symmetric derivative of $f, f_{a p}^{(1)}(x)$.

Received by the editors February 2, 1988.

1980 Mathematics Subject Classification (1985 Revision). Primary 26A24; Secondary 26A48. Presented at the Fifth Annual Auburn Miniconference on Real Analysis April 15, 1988 Sponsored by NSF.

Key words and phrases. Symmetric derivative, approximate symmetric derivative, density, monotonicity. 


\section{A symmetric density property for open sets.}

LEMMA 1. Let $W$ be a measurable subset of $(a, b)$ with $\bar{d}^{+}(W, x) \geq \bar{d}^{-}(W, x)$ and $\underline{d}^{+}(W, x) \geq \underline{d}^{-}(W, x)$ for all $x$ in $(a, b)$. If $d(W, z)=1$ and $d(W, y)=0$ for some $z<y$ and $0<\gamma<1$, then $d(W, x)=\gamma$ for some $x$ between $z$ and $y$.

Proof. Let $f(x)=\int_{a}^{x} \chi_{W}(t) d t$. Then $f$ is continuous on $[a, b]$ and $D^{+} f \geq D^{-} f$ and $D_{+} f \geq D_{-} f$ on $(a, b)$. Suppose $f^{\prime}(z)=1$ and $f^{\prime}(y)=0$ for some $z<y$ and $0<\gamma<1$. Let $g(x)=f(x)-\gamma x$. Then $D^{+} g \geq D^{-} g$ and $D_{+} g \geq D_{-} g$ on $(a, b)$. Since $g^{\prime}(z)=1-\gamma>0$ and $g^{\prime}(y)=-\gamma<0, g$ assumes its maximum on $[z, y]$ at some $x$ in $(z, y)$. Since $D^{+} g(x) \leq 0$ and $D^{-} g(x) \geq 0$, we have $D^{+} g(x)=D^{-} g(x)=0$. Also, $D_{-} g(x)=0$. Finally, $D_{+} g(x) \leq 0$, so $D_{+} g(x)=0$. Thus $g^{\prime}(x)=0$ and $f^{\prime}(x)=\gamma$, so $d(W, x)=\gamma$ as required. This finishes the proof of the lemma.

THEOREM 1. Let $W$ and $B$ be open subsets of $[a, b]$ with $\lambda(W \cup B)=b-a$. If $W B(x)$ has density 0 at 0 for each $x$ in $(a, b)$, then $W B(x)=\varnothing$ for each $x$ in $(a, b)$.

ProOF. Suppose $W B(z) \neq \varnothing$ for some $z$ and $W B(x)$ has density 0 at 0 for all $x$ in $(a, b)$. It is easy to see that the density requirement forces $W \cap B$ to be empty. Adjoin to $W$ any points contained in open intervals in which $W$ has full measure. Do the same for $B$. Clearly, $W$ and $B$ are still open and disjoint, and the density of $W B(x)$ has not been altered. Let $Q=[a, b]-(W \cup B)$, a closed set, and define $C$ to be the set of points in $Q$ that are both right endpoints of components of $B$ and left endpoints of components of $W$. By Lemma 1 , if $x \in W$ and $y \in B$ with $x<y$, then $[x, y] \cap Q$ is uncountable, since the density of $W$ can be strictly between 0 and 1 only at points in $Q$. This says that $Q$ is the disjoint union $P^{\prime} \cup C$, where $P^{\prime}$ is a perfect set. Let $P_{n}=\left\{x \in P^{\prime} \mid \lambda(W B(x) \cap(0, \delta)) / \delta \leq 0.0001\right.$ for all $0<\delta \leq 1 / n\}$. It is easy to see that each $P_{n}$ is closed. Since $P^{\prime}=\bigcup_{n=1}^{\infty} P_{n}$, we can use the Baire Category Theorem to get a subinterval of $P^{\prime}$ contained in some $P_{n}$. Let $P^{\prime \prime}=[c, d] \cap P^{\prime}$ be such a subinterval. By Lemma 1 , we can pick a point, $m$, in $P^{\prime \prime} \cap(c, d)$ so that $d(W, m)=d(B, m)=\frac{1}{2}$. We then pick $\eta>0$ so that $[m-\eta, m+\eta] \subset[c, d]$ and, for any interval $K \subset[m-\eta, m+\eta]$ that contains $m$, $\left[\lambda(W \cap K) / \lambda(K)-\frac{1}{2}\right]<0.00001$.

Let $P=[m-\eta, m+\eta] \cap P^{\prime}$. For ease of calculation and notation, we will assume that $m=\frac{1}{2}$ and $\eta=\frac{3}{2}$. This gives:

(1) $P \subset[-1,2]=I, J=[0,1]$ is the middle third of $I$;

(2) if $x \in P$ and $(x-\delta, x+\delta) \subset I$, then $\lambda(W B(x) \cap(0, \delta)) / \delta \leq 0.0001$;

(3) for any interval $K \subset I$ that contains $\frac{1}{2}$,

$$
\left|\lambda(W \cap K) / \lambda(K)-\frac{1}{2}\right|<0.00001 \text {. }
$$

Let $W \cap(0,1)=\bigcup G_{n}$ and $B \cap(0,1)=\bigcup H_{n}$, the components of $W$ and $B$ in $(0,1)$. If we order the intervals in $\left(\bigcup G_{n}\right) \cup\left(\cup H_{n}\right)$ by decreasing lengths, let $\varepsilon$ be the first length so that the union of intervals of length greater than or equal to $\varepsilon$ has measure greater than or equal to $\frac{1}{8}$. We can assume then, without loss of generality, that $\bigcup\left(G_{n} \mid \lambda\left(G_{n}\right) \geq \varepsilon\right)$ has measure at least $\frac{1}{16}$. Let this collection be $\left\{G_{1}, \ldots, G_{k}\right\}$. Now, renumber these intervals in order from left to right. Let $j$ be largest such that $G_{j}$ is not a subset of the interval $(0.9999,1)$. Then $\lambda\left(\bigcup_{n=1}^{j} G_{n}\right) \geq \frac{1}{16}-0.0001$.

LEMMA 2. If $a, b$ are in $J \cap P, a<b$, and $\lambda(W \cap(a, b)) \geq(b-a) \gamma$, then there is a $c>b$ in $P$ so that $c-a \geq(b-a)(0.9999+\gamma)$ and $\lambda(W \cap(a, c)) \geq(c-a)(\gamma-0.00005)$. 
PROOF. Let $c^{\prime}=2 b-a$. Then $c^{\prime}-a=2(b-a) \geq(b-a)(0.9999+\gamma)$ and $\lambda\left(W \cap\left(b, c^{\prime}\right)\right) \geq\left(c^{\prime}-b\right)(\gamma-0.0001)$ by (2). Thus $\lambda\left(W \cap\left(a, c^{\prime}\right)\right) \geq\left(c^{\prime}-a\right)(\gamma-0.00005)$. We distinguish three cases.

Case (i). If $c^{\prime} \in P$, let $c=c^{\prime}$.

Case (ii). If $c^{\prime} \in W \cup C$, then $c^{\prime}$ is either contained in, or is a left endpoint of, a component of $W$. Let $c$ be the right endpoint of this component, which is a point in $P$. This increases the relative measure of $W$ so that $\lambda(W \cap(a, c)) \geq$ $(c-a)(\gamma-0.00005)$.

Case (iii). If $c^{\prime} \in B$, let $c$ be the left endpoint of the component of $B$ containing $c^{\prime}$, which is in $P$. Since $b \in P$, we have $\left(c^{\prime}-c\right) /\left(c^{\prime}-b\right) \leq 1-(\gamma-0.0001)=1.0001-\gamma$. So, $\left(c^{\prime}-c\right) \leq(1.0001-\gamma)\left(c^{\prime}-b\right)=(1.0001-\gamma)(b-a)$. Then $c-a=\left(c^{\prime}-a\right)-\left(c^{\prime}-c\right) \geq$ $2(b-a)-(1.0001-\gamma)(b-a)=(b-a)(0.9999+\gamma)$. Since $\left(c, c^{\prime}\right) \subset B, \lambda(W \cap(a, c))=$ $\lambda\left(W \cap\left(a, c^{\prime}\right)\right) \geq\left(c^{\prime}-a\right)(\gamma-0.00005)>(c-a)(\gamma-0.00005)$.

This finishes the proof of the lemma.

Let $(a, b)$ be one of the intervals $G_{1}, \ldots, G_{j}$. We will show that seven successive applications of Lemma 2, with the left endpoint a fixed, produces an interval contained in $J$. For $a \leq \frac{1}{2}$, this is easy to see by the fact that $W$ has relative measure roughly $\frac{1}{2}$ on any interval containing $\frac{1}{2}$. Consider $a>\frac{1}{2}$. Suppose $n$ applications of Lemma 2 to $(a, b)$ produces the interval $(a, c)$ in $J$, but Lemma 2 applied to $(a, c)$ extends beyond 1. Recall that $a<0.9999$. Then $\lambda(W \cap(a, c)) \geq(c-a)(1-0.00005 n)$, since $(a, b) \subset W$ and each application of the lemma can reduce the relative measure of $W$ by at most 0.00005 . We also have $\lambda\left(W \cap\left(\frac{1}{2}, a\right)\right) \geq 0.49999\left(a-\frac{1}{2}\right)$ and $\lambda\left(W \cap\left(\frac{1}{2}, c\right)\right) \leq 0.50001\left(c-\frac{1}{2}\right)$ by $(3)$. This gives $\lambda(W \cap(a, c)) \leq 0.50001(c-$ $\left.\frac{1}{2}\right)-0.49999\left(a-\frac{1}{2}\right)=0.50001(c-a)+0.00002\left(a-\frac{1}{2}\right) \leq 0.50001(c-a)+0.00002$. Combining the two inequalities, we get $1-0.00005 n \leq 0.50001+0.00002 /(c-a)$. Since $2 c-a>1$ and $a<0.9999, c-a \geq 0.0001 / 2=0.00005$. Thus $1-0.00005 n \leq$ $0.50001+0.00002 / 0.00005=0.90001$, or $n>1999$.

Now apply Lemma 2 seven times to $G_{1}$ to get the interval $I_{1}$. Take the first $G_{i}$ not in $I_{1}$ and apply Lemma 2 seven times to get the interval $I_{2}$. Repeat this process through $G_{j}$ to produce the intervals $I_{1}, \ldots, I_{m}$. By straightforward calculation, each of these intervals is of length at least $(1.9999)^{7} \varepsilon \geq 64 \varepsilon$ and has relative measure of $W$ at least $1-(0.00005)(7)=0.99965$. Since $\bigcup_{n=1}^{j} G_{n} \subset \bigcup_{n=1}^{m} I_{n}, \bigcup_{n=1}^{m} I_{n}$ has measure at least $\frac{1}{16}-0.0001$.

Let $A=\bigcup_{n=1}^{m} I_{n}$, and $\beta=(B-A) \cap(0,1)$. Observe that $\beta$ is open since no component of $B$ contains an endpoint of any of the finitely many components of $A$. We let $T$ represent the triangle with vertices $(0,0),(1,0)$, and $\left(\frac{1}{2}, \frac{1}{2}\right)$, and $D \subset T$ is $\{(x, y) \mid y \in A \beta(x) \cup \beta A(x)\}$. Let $H_{i}$ and $I_{k}$ be components of $\beta$ and $A$ respectively. By intersecting the lines with slopes \pm 1 through the endpoints of $H_{i}$ and $I_{k}$, we obtain a rectangular region contained in $D$. Inside this region inscribe the parallelogram, $D_{i k}$, with two vertical sides that contain, as opposite vertices, the midpoints of the two shorter sides of the rectangle. Then $D_{i k}$ projects onto the $x$-axis as an interval, $V_{i k}$, of length $\max \left(\lambda\left(H_{i}\right), \lambda\left(I_{k}\right)\right) / 2$, and the vertical section of $D_{i k}$ is of length $\min \left(\lambda\left(H_{i}\right), \lambda\left(I_{k}\right)\right) / 2$. Thus, the area of $D_{i k}$ is equal to $\lambda\left(H_{i}\right) \lambda\left(I_{k}\right) / 4$ and $\lambda\left(V_{i k}\right) \geq \lambda\left(I_{k}\right) / 2 \geq 32 \varepsilon$. The area of $\bigcup D_{i k}$ is then greater than or equal to $\lambda(\beta) \lambda(A) / 4 \geq(0.49999-0.00035)\left(\frac{1}{16}-0.0001\right) / 4 \geq 0.0076$. Let $L_{i k}$ be the left half of $D_{i k}$. Then $\bigcup L_{i k}$ has area at least 0.0038 . 
Since $\bigcup L_{i k} \subset T$, some $z$ in $(0,1)$ must have a vertical section of $\bigcup L_{i k}$ of linear measure at least 0.0038 . Then every $x$ in $(z, z+16 \varepsilon)$ has a vertical section of $\bigcup D_{i k}$ of measure at least 0.0038 also. Let $E=W \cap A$ and $F=A-W$. Then, for each $x$ in $(z, z+16 \varepsilon)$, we have

$$
\begin{aligned}
\lambda(W B(x) \cup B W(x)) & \geq \lambda(E B(x))+\lambda(B E(x)) \\
& \geq \lambda(\{h \mid(x, h) \in D\})-\lambda(F B(x))-\lambda(B F(x)) \\
& \geq 0.0038-2 \lambda(F) \\
& \geq 0.0038-0.00035-0.00035=0.0031 .
\end{aligned}
$$

LEMMA 3. For $x$ in $(0,1)$ and $z=\max (x, 1-x), \lambda(W B(x) \cap(0, z)) \geq$ $\lambda(B W(x) \cap(0, z))-0.00004 z$.

PROOF. We prove the case $z=x \geq \frac{1}{2}$, the other case being symmetric. Let $\mu_{1}, \mu_{2}$, and $\mu_{3}$ be the relative measures of $B$ in the intervals $(x, 2 x),(0, x)$, and $(0,2 x)$ respectively. Then $\mu_{3}=\left(\mu_{2}+\mu_{1}\right) / 2$ gives $\mu_{1}=2 \mu_{3}-\mu_{2} \geq 2(0.49999)-$ $0.50001=0.49997$ by (3). Then $\lambda((W B(x) \cup B B(x)) \cap(0, x))=\lambda(B \cap(x, 2 x)) \geq$ $0.49997 x$. Also, (3) gives $\lambda((W B(x) \cup W W(x)) \cap(0, x))=\lambda(W \cap(0, x)) \geq 0.49999 x$. Adding these two inequalities and using the fact that $W B(x) \cup W W(x) \cup B B(x) \cup$ $B W(x)$ has full measure in $(0, x)$, we get $\lambda(W B(x) \cap(0, x))+x-\lambda(B W(x) \cap(0, x)) \geq$ $0.99996 x$, which is the desired inequality. This finishes the proof of the lemma.

We now establish an estimate for the longest possible component of $W$ or $B$ in $(0,1)$.

LEMMA 4. If $(a, b)$ is a component of $W \cup B$ in $(0,1)$, then $b-a \leq 0.000025$.

ProOF. Since no component can contain $\frac{1}{2}$, it suffices to prove the case $(a, b)$ a component of $W$ contained in $\left(\frac{1}{2}, 1\right)$. Let $\mu_{1}=1, \mu_{2}$, and $\mu_{3}$ be the relative measures of $W$ in the intervals $(a, b),\left(\frac{1}{2}, a\right)$, and $\left(\frac{1}{2}, b\right)$ respectively. Then $\left(b-\frac{1}{2}\right) \mu_{3}=$ $\left(a-\frac{1}{2}\right) \mu_{2}+(b-a)$. Rearrangement gives $(b-a)=\left(a-\frac{1}{2}\right)\left(\mu_{3}-\mu_{2}\right) /\left(1-\mu_{3}\right) \leq$ $0.5(0.00002) /(1-0.50001) \leq 0.000025$. This finishes the proof of the lemma.

Let $c$ be the midpoint of $V=(z, z+16 \varepsilon)$, and $U$ the union of all $G_{n}$ and $H_{n}$ of length greater than $\varepsilon$. Then $\lambda(U)<\frac{1}{8}$. Without loss of generality, we may assume $0<c \leq \frac{1}{2}$. Define $U^{\prime}=\{2 x-c \mid x \in U\}$. Since $\lambda\left(U^{\prime}\right)<\frac{1}{4}, \lambda\left(U \cup U^{\prime}\right)<\frac{3}{8}$. We can then pick $y \in\left[\frac{9}{16}, \frac{15}{16}\right]-\left(U \cup U^{\prime}\right)$. Let $x=(y+c) / 2$. Since $2 x-c=y \notin U^{\prime}$, $x \notin U$. Thus, there are points $s$ and $t$ in $P$ within $\varepsilon$ of $x$ and $y$ respectively. To see this, consider $y$ first. If $y \in P$, let $t=y$. If $y \notin P$, then $y$ is in the closure of some $G_{n}$ or $H_{n}$. By Lemma 4 and the fact that $y \notin U$, this interval can have length no greater than $\varepsilon \leq 0.000025)$. Therefore, both endpoints of this interval are in $(0,1)$ and at least one of them is in $P$ and is designated $t$. The points $x$ and $s$ are handled the same way. Then, for $r=2 s-t$, we have $|r-c|=|(2 s-t)-c|=$ $|2 x+2 s-2 x-y+y-t-c|=|(2 x-y-c)+2(s-x)+(y-t)| \leq 0+2|s-x|+|y-t| \leq 3 \varepsilon$. This gives $r \in V$ and $\lambda\left((W B(r) \cup B W(r)) \cap\left(0, r^{\prime}\right)\right) \geq 0.0031$, where $r^{\prime}=\min (r, 1-r)$.

Our last step is to show that $W B(s)$ or $W B(t)$ violates (2). We define four maps as follows. Let $\rho_{1}(h)=h+s-r$ on $(B W(r) \cup W B(r)) \cap\left(0, r^{\prime}\right), \rho_{2}(h)=h$ on $(B W(r) \cup W B(r)) \cap\left(0, r^{\prime}\right), \rho_{3}(h)=h-s+r$ on $(B W(r) \cup W B(r)) \cap[s-$ $\left.r, r^{\prime}\right)$, and $\rho_{4}(h)=-(h-s+r)$ on $(B W(r) \cup W B(r)) \cap(0, s-r)$. Clearly, each $\rho_{i}$ is one-to-one and measure preserving on its domain. If $h-s+r \geq 0$ then 
$(r, h),\left(s, \rho_{1}(h)\right),\left(t, \rho_{2}(h)\right)$, and $\left(s, \rho_{3}(h)\right)$ are the vertices of a square. If $h-s+r<0$, the fourth vertex $(s, h-s+r)$ is reflected above the $x$-axis to $\left(s, \rho_{4}(h)\right)$.

Consider $h \in B W(r)$. Then $\rho_{1}(h) \in B W(s), \rho_{2}(h) \in W B(t), \rho_{3}(h) \in B W(s)$, $\rho_{4}(h) \in W B(s), \rho_{1}(h) \in B Q(s)$, or $\rho_{2}(h) \in Q B(t)$. A similar statement corresponds to $h \in W B(r)$. For $r^{\prime}=\min (r, 1-r), s^{\prime \prime}=\max (s, 1-s)$, and $t^{\prime \prime}=\max (t, 1-t)$, define the following subsets of $(B W(r) \cup W B(r)) \cap\left(0, r^{\prime}\right)$. Let

$$
\begin{aligned}
& S_{1}=\left\{h \mid \rho_{1}(h) \in(B W(s) \cup W B(s)) \cap\left(0, s^{\prime \prime}\right)\right\}, \\
& S_{2}=\left\{h \mid \rho_{2}(h) \in(B W(t) \cup W B(t)) \cap\left(0, t^{\prime \prime}\right)\right\}, \\
& S_{3}=\left\{h \mid \rho_{3}(h) \in(B W(s) \cup W B(s)) \cap\left(0, s^{\prime \prime}\right)\right\}, \\
& S_{4}=\left\{h \mid \rho_{4}(h) \in(B W(s) \cup W B(s)) \cap\left(0, s^{\prime \prime}\right)\right\}, \\
& S_{5}=\left\{h \mid \rho_{1}(h) \in B Q(s) \cup W Q(s) \text { or } \rho_{2}(h) \in Q B(t) \cup Q W(t)\right\} .
\end{aligned}
$$

Since $\lambda(Q)=0$, we have $\lambda\left(S_{5}\right)=0$. Since $(B W(r) \cup W B(r)) \cap\left(0, r^{\prime}\right)=\bigcup_{i=1}^{5} S_{i}$, one of the sets $S_{1}, S_{2}, S_{3}$, or $S_{4}$ has measure at least $0.0031 / 4>0.00075$. Therefore, one of $(B W(s) \cup W B(s)) \cap\left(0, s^{\prime \prime}\right)$ or $(B W(t) \cup W B(t)) \cap\left(0, t^{\prime \prime}\right)$ has measure at least 0.00075 . We assume the former set has measure at least $0.00075 \geq 0.00075 s^{\prime \prime}$.

By (2), $\lambda\left(W B(s) \cap\left(0, s^{\prime \prime}\right)\right) \leq 0.0001 s^{\prime \prime}$, so $\lambda\left(B W(s) \cap\left(0, s^{\prime \prime}\right)\right) \geq 0.00065 s^{\prime \prime}$. But then Lemma 3 gives $\lambda\left(W B(s) \cap\left(0, s^{\prime \prime}\right)\right) \geq 0.00065 s^{\prime \prime}-0.00004 s^{\prime \prime}=0.00061 s^{\prime \prime}$, contradicting (2). This finishes the proof of the theorem.

\section{An application to the approximate symmetric derivative.}

THEOREM 2. If $f$ is continuous on $[a, b]$ and $\underline{f}_{a p}^{(1)} \geq 0$ on $(a, b)$, then $f$ is nondecreasing on $[a, b]$.

PROOF. For $\varepsilon>0$, let $g(x)=f(x)+\varepsilon x$. Then $\underline{g}_{a p}^{(1)}>0$ on $(a, b)$. Let $y$ be any number in the interior of the range of $g$. It is easy to see that $W=$ $g^{-1}(y, \infty)$ and $B=g^{-1}(-\infty, y)$ satisfy the hypotheses of Theorem 1 . Then $g$ must be nondecreasing, since if $c<d$ and $g(c)>g(d)$, we can pick any $y$ between $g(c)$ and $g(d)$ and get $W B((c+d) / 2) \neq \varnothing$, contradicting Theorem 1. Since $\varepsilon$ is arbitrary, $f$ is nondecreasing. This finishes the proof of the theorem.

\section{BIBLIOGRAPHY}

1. N. K. Kundu, On the approximate symmetric derivative, Colloq. Math. 28 (1973), 275-285.

2. L. Larson, Symmetric real analysis: A survey, Real Anal. Exchange 9 (1983-1984), 154-178.

3. $\ldots$, Monotonicity and the approximate symmetric derivative, Real Anal. Exchange 12 (19861987), 121-123.

4. J. Matousek, Approximate symmetric derivatives and monotonicity, Comment Math. Univ. Carolin. 27 (1986), 83-86.

5. S. N. Mukhopadhyay, On approximate Schwarz differentiability, Monatsh. Math. 70 (1966), 454-460.

6. H. W. Pu and H. H. Pu, On approximate Schwarz derivatives, Rev. Roumaine Math. Pures Appl. 25 (1980), 257-264.

Department of Mathematics, California State University, SAN Bernardino, CALIFORNIA 92407 